\title{
Editorial: Responding to the needs of doctoral researchers
}

\author{
Nicola Grayson \\ University of Manchester
}

The challenges faced by doctoral students during the course of their studies are well documented, particularly in relation to issues of isolation, wellbeing and mental health. These issues were further exacerbated by the pandemic and the subsequent lockdowns. Many researchers felt adrift from their usual support networks and had to motivate themselves to write whilst grappling with caring responsibilities, having to alter the scope of their research, worrying about future prospects, and of course, coping with the loss of loved ones.

This section of the Compendium presents two key perspectives on supporting doctoral students, which confirm and enhance one another. The first three papers set out how those who support researchers adapted their content and approach to take into account the specific needs of their audience in relation to the pandemic. We see commonalities here in terms of creating and emphasising an all-important sense of community. Those supporting researchers took bold steps to advocate for their audience, providing online spaces for them to connect with one another to share their experiences, to reflect on their writing, and to feel less alone.

You will read about how live, synchronous Shut Up and Write sessions supported doctoral students to carve out time to write whilst anchoring this support to asynchronous materials that could be accessed anytime and from anywhere. You will read how a self-employed academic writing teacher for doctoral students in Germany and neighbouring countries sought to replicate a tried and tested hands-on, analogue approach to keep participants engaged and add an extra dimension of reality to online support, which sought to recreate and connect researchers with the face to face support they missed. You will also read of how those enrolled on a Professional Doctorate in Education were provided with online spaces which allowed them to create cross-cohort and transnational groups, and how they formed supportive subgroups in addition to those facilitated by their learning providers. 
You will read about the importance of simple conversations for connecting researchers with their peers as their identity begins to shift from student to teacher. To confirm and validate some of the approaches set out by support providers, you will then read accounts written by doctoral students themselves. The first of these communicates the impact the pandemic had on the researcher's all important relationship with their supervisor, it sets out how they overcame the challenges of online, asynchronous communication and how, by reaching out to peers and seeking creative outlets, they got the support they needed to keep going. Finally, you will read about the challenges faced by a doctoral student in Zimbabwe and gain insight into how the challenges of the pandemic impacted researchers in a developing country where the digital infrastructure of HE institutions could not cope as effectively with the 'online pivot'. You will hear how this researcher sought to cope with the challenges of working on an area that was subsumed by the pandemic and the role that academic networks and online writing support played in supporting this student to make a bold transition to a new institution in order to complete their studies.

Overall, the papers in this section provide a fascinating insight into the world of postgraduate researchers and the impact the pandemic had on those who support them. They demonstrate that everything from doctoral students' ability to work on their projects, maintain productivity and sustain good working habits, as well stay connected to their networks and institutions was affected, but the authors' creative solutions and their ability to see the silver lining generated solutions that may even be extended beyond the emergency response. 